\title{
Obesity and Periodontitis: A Puzzle Yet to be Completed
}

\author{
Sania*, Sukanya Mohanty, Alladi Vaishnavi and Aparimita saxena \\ Department of Periodontics, Kothiwal Dental College \& Research Centre, India
}

Submission: May 12, 2017;Published: July 14, 2017

*Corresponding author: Sania, Department of Periodontics, Kothiwal Dental College \& Research Centre, UP, India, Email: saniabds@gmail.com

\begin{abstract}
Obesity is affecting as a worldwide diseases for whom diagnostics have yet not been perceived. With every growing year, the prevalence and incidence is increasing. Obesity has increased in prevalence and is a major contributor to worldwide morbidity. It has been a recognized as a major public health problem and evidence exists of its role as a major risk factor for a number of conditions, such as cardiovascular diseases, diabetes mellitus, cancer, osteoarthritis and gall bladder disease. One consequence of obesity is an increased risk for periodontal disease, although periodontal inflammation might, in turn, exacerbate the metabolic syndrome of which obesity is one component.
\end{abstract}

\section{Introduction}

Obesity can be seen as the first tide of a defined clus ᄀter of non-communicable diseases called "New World Synᄀdrome," creating an enormous socioeconomic and public health burden in poorer countries. The World Health Organization has described obesity as one of today's most neglected public health problems, affecting every region of the globe [1]. In India, obesity is emerging as an important health problem particularly in urban areas. Almost $30-65 \%$ of adult urban Indians are either overweight or obese [2]. Periodontal disease is an entity of localized infections that involve tooth supporting tissues, the structures that make up the periodontium (i.e., gingiva, periodontal ligament, root cementum, and alveolar bone). In periodontitis, there is destruction of the connective tissue of the tooth attachment apparatus accompanied by apical migration of the apparatus and eventual tooth loss.

It has been suggested that obesity contributes to an overall systemic inflammatory state through its effect on metabolic and immune parameters; thereby increasing susceptibility to periodontal disease (Lacopino AM, 2009). Adipose tissue secretes several cytokines and hormones that are involved in inflammatory processes, suggesting that similar pathways are involved in the pathophysiology of obesity and periodontitis $[3,4]$. Obesity is an excess amount of body fat in proportion to lean body mass, to the extent that health is impaired. Obesity is defined based on body mass index (BMI), also called Quetelet Index, which is the ratio of body weight (in $\mathrm{kg}$ ) to body height squared (in $\mathrm{m}^{2}$ ). BMI is highly correlat $\urcorner$ ed with fat mass and morbidity and mortality and therefore sufficiently reflects obesity-related disease risk in a wide range of populations. The World Health Organization and the National Heart, Lung and Blood Institute (NHLBI) define overweight as a body mass index of 25-29.9 and obesity as a body mass index of $\geq 30$ [5].

\section{Obesity-related diseases}

Hypertension: Most important determinants of elevated blood pressure levels have been identified as Overweight and obesity. Weight gain is consistently associated with increased blood pressure, and that weight loss decreases blood pressure independent of changes in sodium intake. Compared with normal-weight individuals, obese persons have an up to 5 times higher risk of hypertension, and up to two thirds of cases of hypertension can be attributed to excess weight. The Mechanisms of obesity-related hypertension include a wide range like increased sympathetic nerve activity, sodium and volume retention, renal abnormalities, insulin resistance, hyperleptinemia and increased secretion of angiotensinogen from adipocytes [6].

Type 2 diabetes: Obesity and type 2 diabetes relationship is particularly close. Obese persons have a more than 10fold increased risk of developing type 2 diabetes compared with normal-weight persons. Type 2 diabetes actors, includes lipotoxicity and glucose toxicity, as well as obesity-derived cytokines [7]. 
Cardiovascular disease: Obese persons have an about 1.5-fold increased risk for cardiovascular disease (including coronary heart disease and cerebrovascular disease). Obesity is also associated with an about 2 -fold higher risk of heart failure and a $50 \%$ increased risk of atrial fibrillation $[8,9]$.

Osteoarthritis: Obesity is associated with bone knee and hip arthritis, and with arthritis involving the carpometacarpal joints of the hand. Recent studies have proved that being overweight antedates the development of knee osteoarthritis and increases the risk of radiographic progression [10].

Respiratory disorders: Visceral fat accumulation results in restrictive respiratory function with reduced forced vital capacity and expiratory reserve volume. Obesity is the major reversible risk factor for obstructive sleep apnea syndrome. The prevalence rises from $2 \%$ to $4 \%$ in the general population to a prevalence of at least $40 \%$ in morbidly obese patients. Orofacial findings of this syndrome includes a retrognathic mandible, narrow palate, large neck circumference, long soft palate, tonsillar hypertrophy, nasal septal deviation and relative macroglossia. Waist circumference tends to be a better predictor of this syndrome than body mass index $[10,11]$.

Metabolic syndrome: The metabolic syndrome is a concept that encompasses metabolic abnormalities that co-occur to a greater degree than would be expected by chance alone, and which predisposes individuals at a high risk to develop cardiovascular disease. Although the exact underlying cause of metabolic syndrome is unknown, the more recent definitions emphasize the focus on abdominal obesity as its core component (International Diabetes Federation, 2005). This approach is supported by a growing number of studies showing that the adipose tissue itself has the capability of producing several hormones and proteins, which are involved in the development of obesity-related diseases $[11,12]$.

Association between obesity and periodontal disease: Obesity is second only to smoking as the strongest risk factor for inflammatory periodontal tissue destruction [13]. The first report on the relationship between obesity and periodontal disease appeared in 1977, when Perlstein et al. [13] observed histopathologic changes in the periodontium in hereditary obese Zucker rats. Using ligature-induced periodontitis, they found alveolar bone resorption to be greater in obese animals compared with non-obese rats. Also, it seemed that under healthy oral conditions, obesity does not promote pathologic periodontal alterations; however, in response to bacterial plaque accumulation, periodontal inflammation and destruction were more severe in obese animals [14]. In 1998, Saito et al. analyzed 241 healthy Japanese individuals and showed, for the first time, an association between obesity and periodontal disease in humans. In addition, studies have indicated that the fat distribution pattern plays a crucial role in the association with periodontitis $[15,16]$. Another recent study by Saito et al. concluded that obesity is associated with deep periodontal pockets, independent of glucose tolerance status. The underlying biological mechanisms for the association of obesity with periodontitis are not well known; however, adipose-tissuederived cytokines and hormones may play a key role. Fat tissue is not merely a passive triglyceride reservoir of the body, but also produces a vast amount of cytokines and hormones, collectively called adipokines or adipocytokines, which in turn may modulate periodontitis [17].

Adipose-tissue-derived hormones and cytokines (adipokines) inflammatory markers: Adipose tissue secretes proinflammatory cytokines such as tumor necrosis factor-alpha (TNF- $\alpha$ ) and interleukin-6 (IL-6). TNF- $\alpha$ and IL- 6 are the main inducers of acute-phase hepatic protein production, including C-reactive protein (CRP). Adipokines play an important role:

Adipokines play a number of different roles such as

a. Hormone-like proteins, e.g. Leptin and adiponectin

b. Classical cytokines, e.g. TNF- $\alpha$ and IL-6

c. Protein involved in vascular haemostasis, e.g. Plasminogen activator inhibitor-1, tissue factor

d. Promoters of angiogenesis, e.g. Vascular endothelial growth factor

e. Acute phase respondents, e.g. CRP

Leptin: Leptin is a pleiotropic cytokine, secreted by adipocytes, involved in a variety of biological processes, including energy metabolism, endocrine functions, reproduction and immunity. Leptin is thought to act as a "lipostat" that regulates adipose tissue mass. As a negative feedback mechanism, elevated leptin concentrations result in increased energy expenditure, decreased food intake and a negative energy balance. In contrast, most overweight and obese persons show resistance to leptin at the receptor level and therefore have higher leptin levels than non-overweight individuals.10leptin has also been shown to be involved in bone metabolism [18].

Adiponectin, and other adipose-tissue-derived cytokines: Adiponectin is a circulating hormone secreted by adipose tissue that is involved in glucose and lipid metabolism and which accounts for about $0.05 \%$ of total serum proteins. Adiponectin improves insulin sensitivity and have antiatherogenic and anti-inflammatory properties, and low plasma adiponectin levels have been shown to predict type 2 diabetes and coronary heart disease in humans $[19,20]$. As more and more adipose-tissue-derived cytokines and hormones are being discovered, the complexity of the endocrine network of which these mediators are a part becomes more and more apparent. Recent additions to this list of adipokines include visfatin, which elicits insulin-like effects, and serum-retinol-binding protein 4 (RBP4). Regarded initially as markers mainly related to weight regulation and insulin resistance, it has become clear that hormones like leptin, resist in or adiponectin are involved 
in a variety of functions and diseases, including cardiovascular disease, diabetes and inflammatory diseases [21].

Association of periodontitis with obesity-related chronic diseases: Fat cells were once thought of as having limited function energy storage. It is now known that fat cells produce many chemical signals and hormones. Many of these substances are thought to increase overall inflammation in the body. This may lead to decreased immune status, which increases susceptibly to periodontal disease. The inflammation may also decrease blood flow to the gums and cause disease progression. Proinflammatory cytokines play an important role in close relationship among periodontitis, obesity and chronic diseases. In fact, this association may be multidirectional. For example, it has been well established that inflammation is an essential component in the development of atherosclerosis, and observational studies showed that periodontitis is associated with a moderately, but significantly, higher risk of coronary heart disease [22-24]. Interventional studies that examined the effects of antibiotic treatment on cardiovascular risk have generally failed to show any beneficial effect; however, these studies have mostly been of short duration (less than 1 year of treatment) and have investigated the effects on secondary prevention only. Inflammatory diseases like periodontitis induce the production of proinflammatory cytokines such as TNF- $\alpha$, IL-1 and IL-6 [22]. It has been suggested that the secretion of TNF- $\alpha$ by adipose tissue triggered by LPS from periodontal gram-negative bacteria promotes hepatic dyslipidemia and decreases insulin.

Type 2 diabetes and decreased insulin sensitivity are associated with the production of advanced glycation end products (AGE), which trigger inflammatory cytokine production, thus predisposing to inflammatory diseases such as periodontitis. These observations suggest a potential interaction among obesity, periodontitis and chronic disease incidence, although present studies are insufficient to conclude whether such associations are causal. Thus in addition to being a risk factor for type 2 diabetes and coronary heart disease, obesity-related inflammation may also promote periodontitis. Conversely, periodontitis, once it exists, may promote systemic inflammation and thereby increase the risk of coronary heart disease. In this context, it is interesting to note that periodontal treatment has been shown to reduce circulating TNF- $\alpha$ and serum levels of glycosylated hemoglobin, and has beneficial effects on the control of type 2 diabetes [25].

Assessment in dental practice: Obesity has taken on epidemic proportions internationally. Much comorbidity is associated with obesity and has con $\neg$ sequences for oral health professionals It has been suggested that supine patient positioning should be avoided, to maximize the pulmonary mechanics. Impaired chest expansion decreases vital capacity and tidal function, which compromises tissue oxygenation. These conditions put the obese person at high anesthetic and surgical risk. Wound-healing processes are dependent on sufficient tissue oxygenation. Also, higher incidences of infections and post-surgical hematoma formation have been reported among obese persons. The vulnerability to wound complications increases morbidity and mortality of obese persons. Pharmacological aspects, such as altered pharmacokinetics due to the person's increased blood volume or fat mass and technical incompatibilities, such as small dental chairs or tight blood pressure cuffs, should be considered. Also, a close collaboration with the general physician and the dietician may be beneficial to ensure effective periodontal treatment

\section{Conclusion}

Obesity is a complex disease, and its relationship to oral status has been realized by the scientific community in recent years. Periodontists must be aware of the increasing numbers of obese persons and of the significance of obesity as a multiple-risk-factor syndrome for overall and oral health. Proinflammatory cytokines may be a multidirectional link among periodontitis, obesity and other chronic diseases. Both obesity and periodontal disease significantly impact an individual's overall health Understanding the relationship between obesity and the risk factors that lead to periodontal disease is very important. With the increasing rate of child and adolescent obesity, the prevalence of periodontal disease will follow. Keys to prevention of periodontal disease are through proper oral hygiene, a balanced diet and routine dental visits. Although this relationship needs further investigation, periodontists should counsel obese persons regarding the possible oral complications of obesity, to diminish the future risk of occurrence for these individuals.

\section{References}

1. Mohan Reddy N, Kalyana Kumar Ch, Jamil K (2012) New World Syndrome (Obesity) in South India 1: 567.

2. Giri DK, Kundapur PP, Bhat GS, Bhat KM, Guddattu V (2013) Periodontal disease and obesity in an Indian population. Nepal Journal of Medical Sciences 2(2): 144-149.

3. Pischon N, Heng N, Bernimoulin JP, Kleber BM, Willich SN, et al. (2007) Obesity, Inflammation, and Periodontal Disease. J Dent Res 86(5): 400409.

4. Lacopino AM (2009) Concepts in Oral Systemic health. Journal of the Canadian Dental Association 75(2): 92-93.

5. Must A, Spadano J, Coakley EH, Field AE, Colditz G, et al. (1999) The disease burden associated with overweight and obesity. JAMA 282(16): 1523-1529.

6. Genco RJ, Grossi SG, Ho A, Nishimura F, Murayama Y (2005) A proposed model linking inflammation to obesity, diabetes, and periodontal infections. J Periodontol 76(11): 2075-2084.

7. Pouliot MC, Després JP, Lemieux S, Moorjani S, Bouchard C, et al. (1994) Waist circumference and abdominal sagittal diameter: best simple anthropometric indexes of abdominal visceral adipose tissue accumulation and related cardiovascular risk in men and women. Am J Cardiol 73(7): 460-468.

8. Wilson PW, D’Agostino RB, Sullivan L, Parise H, Kannel WB (2002) Overweight and obesity as determinants of cardiovascular risk: the Framingham experience. Arch Intern Med 162(16): 1867-1872. 


\section{Current Research in Diabetes \& Obesity Journal}

9. Jagannathachary S, Kamaraj D (2007) Obesity and periodontal disease. J Indian Soc Periodontol 14(2): 96-100.

10. Magliocca KR, Helman JI (2005) Obstructive sleep apnea: diagnosis, medical management and dental implications. J Am Dent Assoc 136(8): 1121-1129.

11. Eckel RH, Alberti KG, Grundy SM, Zimmet PZ (2010) The metabolic syndrome. Lancet 375(9710): 181-183.

12. Nishida N, Tanaka M, Hayashi N, Nagata H, Takeshita T, et al. (2005) Determination of smoking and obesity as periodontitis risks using the classification and regression tree method. J Periodontol 76(6): 923928

13. Perlstein MI, Bissada NF (1977) Influence of obesity and hypertension on the severity of periodontitis in rats. Oral Surg Oral Med Oral Pathol 43(5): 707-719.

14. Saito T, Shimazaki Y, Kiyohara Y, Kato I, Kubo M, et al. (2005) Relationship between obesity, glucose tolerance, and periodontal disease in Japanese women: the Hisayama study. J Periodontal Res 40(4): 346-353.

15. Wood N, Johnson RB, Streckfus CF (2004) Comparison of body composition and periodontal disease using nutritional assessment techniques: Third National Health and Nutrition Examination Survey (NHANES III). J Clin Periodontol 30:321-327.

16. Kershaw EE, Flier JS (2004) Adipose tissue as an endocrine organ. J Clin Endocrinol Metab 89(6): 2548-2556.

17. Thomas T, Gori F, Khosla S, Jensen MD, Burguera B, et al. (1999) Leptin acts on human marrow stromal cells to enhance differentiation to osteoblasts and to inhibit differentiation to adipocytes. Endocrinology 140(4): 1630-1638.

18. Lindsay RS, Funahashi T, Hanson RL, Matsuzawa Y, Tanaka S, et al. (2002) Adiponectin and development of type 2 diabetes in the Pima Indian population. Lancet 360(9326): 57-58.

19. Pischon T, Girman CJ, Hotamisligil GS, Rifai N, Hu FB, et al. (2004) Plasma adiponectin levels and risk of myocardial infarction in men. JAMA 291(14): 1730-1737.

20. Pischon N, Heng N, Bernimoulin JP, Kleber BM, Willich SN, et al. (2007) Obesity, inflammation, and periodontal disease. J Dent Res 86(5): 400409.

21. Beck JD, Offenbacher S (2005) Systemic effects of periodontitis: epidemiology of periodontal disease and cardiovascular disease. J Periodontol 76(11): 2089-2100.

22. Dietrich T, Garcia RI (2005) Associations between periodontal disease and systemic disease: evaluating the strength of the evidence. Periodontol 76(11): 2175-2184.

23. Mattila KJ, Pussinen PJ, Paju S (2005) Dental infections and cardiovascular diseases: a review. J Periodontol 76(11): 2085-2088.

24. Nishimura F, Iwamoto Y, Mineshiba J, Shimizu A, Soga Y, et al. (2003) Periodontal disease and diabetes mellitus: the role of tumor necrosis factor-alpha in a 2-way relationship. J Periodontol 74(1): 97-102.

25. Kempers KG, Foote JW, DiFlorio-Brennan T (2000) Obesity: prevalence and considerations in oral and maxillofacial surgery. J Oral Maxillofac Surg 58(2): 137-143.

\section{Your next submission with Juniper Publishers} will reach you the below assets

- Quality Editorial service

- Swift Peer Review

- Reprints availability

- E-prints Service

- Manuscript Podcast for convenient understanding

- Global attainment for your research

- Manuscript accessibility in different formats

( Pdf, E-pub, Full Text, Audio)

- Unceasing customer service

Track the below URL for one-step submission https://juniperpublishers.com/online-submission.php 